\title{
Genetic Stratification in Myeloid Diseases: From Risk Assessment to Clinical Decision Support Tool
}

\author{
Yishai Ofran, M.D. ${ }^{1,2}{ }^{2}$ \\ ${ }^{\prime}$ Department of Hematology and Bone Marrow Transplantation, Rambam Health Care Campus, Haifa, \\ Israel; and ${ }^{2}$ Bruce Rappaport Faculty of Medicine, Technion, Israel Institute of Technology, Haifa, Israel
}

\begin{abstract}
Genetic aberrations have become a dominant factor in the stratification of myeloid malignancies. Cytogenetic and a few mutation studies are the backbone of risk assessment models of myeloid malignancies which are a major consideration in clinical decisions, especially patient assignment for allogeneic stem cell transplantation. Progress in our understanding of the genetic basis of the pathogenesis of myeloid malignancies and the growing capabilities of mass sequencing may add new roles for the clinical usage of genetic data. A few recently identified mutations recognized to be associated with specific diseases or clinical scenarios may soon become part of the diagnostic criteria of such conditions. Mutational studies may also advance our capabilities for a more efficient patient selection process, assigning the most effective therapy at the best timing for each patient. The clinical utility of genetic data is anticipated to advance further with the adoption of deep sequencing and next-generation sequencing techniques. We herein suggest some future potential applications of sequential genetic data to identify pending deteriorations at time points which are the best for aggressive interventions such as allogeneic stem cell transplantation. Genetics is moving from being mostly a prognostic factor to becoming a multitasking decision support tool for hematologists. Physicians must pay attention to advances in molecular hematology as it will soon be accessible and influential for most of our patients.
\end{abstract}

\footnotetext{
Abbreviations: allo-HCT, allogeneic hematopoietic cell transplantation; AML, acute myeloid leukemia; APL, acute promyelocytic leukemia; CLL, chronic lymphocytic leukemia; CML, chronic myeloid leukemia; CMML, chronic myelomonocytic leukemia; IPSS, International Prognostic Scoring System; MDS, myelodysplastic syndrome; MPN, myeloproliferative neoplasm; MRD, minimal residual disease; NGS, next-generation sequencing; PMF, primary myelofibrosis; R-IPSS, Revised International Prognostic Scoring System.
}

Citation: Ofran Y. Genetic Stratification in Myeloid Diseases: From Risk Assessment to Clinical Decision Support Tool. Rambam Maimonides Med J 2014;5 (4):eo025. doi:10.5041/RMMJ.10159

Copyright: (C) 2014 Ofran. This is an open-access article. All its content, except where otherwise noted, is distributed under the terms of the Creative Commons Attribution License (http://creativecommons.org/licenses/by/3.0), which permits unrestricted use, distribution, and reproduction in any medium, provided the original work is properly cited.

Conflict of interest: No potential conflict of interest relevant to this article was reported.

*E-mail: y_ofran@rambam.health.gov.il 
KEY WORDS: Acute myeloid leukemia, allogeneic hematopoietic cell transplantation, genetic aberrations, minimal residual disease, myelodysplastic syndrome

\section{INTRODUCTION}

Forty-one years ago Janet Rowley was the first to describe chromosomal translocation in leukemia. ${ }^{1}$ Back in the 1970s, the concept that genetic aberrations may be involved in cancer pathobiology was so revolutionary that her landmark report of $\mathrm{t}(8: 21)$ in acute myeloid leukemia (AML) was rejected by the New England Journal of Medicine because reviewers thought her findings were not important. ${ }^{2}$ Soon afterwards, the value of her findings was recognized, and her second report regarding t(9:22) in chronic myeloid leukemia (CML) was accepted by Nature. ${ }^{3}$ During the last decades, scientists, oncologists, and hematologists have come a long way advancing our knowledge and understanding of the roles of genetic aberrations in cancer pathobiology. Numerous genetic aberrations were identified in each cancerous disease. A current clinical challenge is to pick up aberrations that matter out of a huge haystack of genetic information. Doctors and health care providers are under continuous pressure to catch up with cutting-edge discoveries. The enormous number of possible combinations of coexisting mutations and the growing number of novel available therapies make clinical implications of genetic data complicated. As in many other fields of life, the public is a step ahead of governmental or authorized health care organizations. It is common that patients initiate additional comprehensive genetic testing, expecting their treating physicians to be equipped enough to interpret such results. Unfortunately, most identified mutations are either clinically meaningless, or their value in each clinical setting is still to be recognized. In the following review an up-to-date comprehensive approach for genetic stratification in myeloid diseases will be discussed in light of common clinical dilemmas.

\section{GENETIC CONSIDERATIONS IN THE EVALUATION OF MYELOID DISEASES}

\section{Genetics as a Supportive Tool for Accurate Diagnosis of Myeloproliferative Disorders, Myelodysplasia, and Neoplasms}

Back in 1953 a pre-leukemic state was described in a series of 12 patients, 4 yet a clear pathological definition, classification, and naming the syndrome as myelodysplastic were established only in 1982 by the French-American-British (FAB) group. 5 During the 32 years that followed the FAB classification, myelodysplastic syndrome (MDS) was reclassified twice, 6,7 based on morphology. Currently isolated deletion of $5 \mathrm{q}$ is the only genetic aberration that has implication for MDS diagnosis and classification.

The torrent of novel genetic information is not yet reflected in the way MDS is being diagnosed and classified. The limitations of morphologic classification lead to the recognition of ill-defined entities.7 There is an unmet need for laboratory-supportive tools for the diagnosis and classification of morphologically challenging entities. ${ }^{8,9}$ The potential of genetic data that can support current diagnostic tools is of special importance when morphologic diagnosis is difficult, inconclusive, or nonreproducible by different morphologists. Newly identified driver mutations are found to be associated with otherwise poorly defined diseases and are likely to be included in future MDS or myeloproliferative neoplasm (MPN) classification. Examples for suggested driver mutations are the SET-binding protein 1 (SETBP1) in atypical chronic myeloid leukemia (aCML), ${ }^{10,11} S R S F 2$ in chronic myelomonocytic leukemia (CMML), ${ }^{12-14}$ and $C_{3} F_{3} R$ in chronic neutrophilic leukemia. ${ }^{15,16}$ Another common diagnostic difficulty that can now be overcome using genetic information is the differentiation between primary myelofibrosis and other causes of bone marrow fibrosis. Either calreticulin (CALR) mutation or JAK2 mutation is revealed in most cases of primary myelofibrosis. ${ }^{17}$ It is therefore reasonable to anticipate incorporation of these mutations into future diagnostic and classification criteria. ${ }^{18,19}$

In low-risk MDS, criteria for quantitative enumeration of ring sideroblasts are available, ${ }^{20}$ but the techniques require well-trained morphologists and are time-consuming. One may consider that since the $S F 3 B 1$ mutation has a positive predictive value for the presence of ring sideroblasts of $97.7 \%,{ }^{21}$ this mutation will soon be recognized as supportive to the diagnosis of refractory anemia with ring sideroblasts. Chronic myelomonocytic leukemia (CMML) is currently classified, based on the level of the white blood cell count, into myelodysplastic and myeloproliferative subcategories. It was 
recently suggested that such sub-classification could be better defined by specific genetic profiles. ${ }^{22,23}$

Understanding of the genetic basis of myeloid malignancies will inevitably lead in the near future to the incorporation of some of the abovementioned genetic tests into the working schemes for hematologic diagnosis. Potentially, the identification of pre-leukemic mutations in $\mathrm{AML}^{24}$ may be the first step towards accomplishing a more challenging task, that of determining the boundaries between high-risk MDS or MPN and AML. In the future, genetics may enable molecular identification of patients in whom leukemic transformation would ensue prior to the appearance of traditional clinical and laboratory signs and symptoms.

\section{Prognostication: Cytogenetics and Beyond}

For many years, cytogenetics has been the only genetic information that influenced clinical decisions in MDS. The international prognostic scores both in their primary and revised versions (IPSS and R-IPSS) ${ }^{25,26}$ consider only patients who received supportive therapy and describe the natural history of the disease providing valuable predictions of prognosis. Recent large-scale, comprehensive genetic studies have provided description of the mutation landscape of MDS, ${ }^{27-29}$ identification of new mechanisms involved in disease pathogenesis, ${ }^{30-32}$ and confirmed that MDS is a stem cell disorder. ${ }^{18}$ Results of such exciting scientific progress are still waiting to be incorporated into daily clinical practice.

Unlike MDS, where cytogenetics allows segregation of patients into categories with a well-defined predicted outcome, 33 the prognostic value of cytogenetics is much less prominent in CMML ${ }^{34-36}$ and primary myelofibrosis (PMF). ${ }^{37,38}$ In the absence of a strong cytogenetic influence, the prognostic value of specific mutations is recognized in these diseases. It is not clear whether these mutations have no effect in MDS or their prognostic value in this disease is masked by a strong cytogenetic influence. The ASXL1 mutation is most dominant in CMML 39,40 and PMF, ${ }^{41,42}$ while mutations in other genes may also affect patient prognosis. The interplay between cytogenetic profile and prognostic power of specific mutations is highlighted by the finding that most MDS patients presenting with a complex karyotype also carry a mutation or a deletion in the wellknown tumor suppresser gene TP53.43,44 Whether the aberrant karyotype or the TP53 mutation is responsible for the poor outcome of affected patients is still unknown.

The more we know about the genomic landscape of myeloid malignancies, the more complicated become the combinatorics of potential aberrations to be integrated for prediction of the outcome for a specific patient. Cytogenetics, mutations, microdeletions, methylation abnormalities, and microRNA expression levels are all important players determining patient prognosis. It will therefore be challenging to create an ultimate prognostic model which will be much better than those available today. Physicians should focus on genetic aberrations that can influence therapeutic decisions in every given clinical situation. Examples and suggestions for such an approach are reviewed herein.

\section{THERAPEUTIC DECISIONS IN THE ERA OF MULTIPLE TREATMENT OPTIONS}

In previous years, allogeneic hematopoietic stem cell transplantation (allo-HCT) was the only diseasemodifying therapy available for MDS. Allo-HCT is indicated whenever the risk of death or leukemic transformation is projected to be lower following transplant than the equivalent risk anticipated by the Revised International Prognostic Scoring System (R-IPSS). 45 The International Prognostic Scoring System (IPSS) and R-IPSS considered only patients who received no other therapy than supportive care, but nowadays, due to a tremendous progress in the area of MDS, most patients are candidates for novel disease-modifying drugs (e.g. azacitidine 46,47 and/or lenalidomide ${ }^{4-50}$ ). Retrospective comparisons suggest that allo-HCT may be superior to azacitidine in selected high-risk populations.51-53 However, patients' specific consultation must be outlined with caution, because such retrospective data are subject to inherent biases.54 Allo-HCT may not be suitable for many AML/MDS patients presenting at older age and with co-morbidities. 55-59 However, with modern reduced-intensity conditioning and supportive care, a large proportion of patients with myeloid diseases are considered fit and can survive through transplant. Many of these potential candidates for alloHCT are classified as intermediate-risk, and, even when a survival benefit with allo-HCT compared to supportive care is predicted by R-IPSS, the actual clinical debate is whether allo-HCT will lead to an outcome better than that provided by novel therapies. Notably, patients may prefer hypomethylation therapy over transplant in fear of hampered posttransplant quality of life. 
Novel Agents and Associated Dilemmas in Acute and Chronic Leukemia

During the last decades, targeted therapies with tyrosine kinase inhibitors and all trans-retinoic acid have revolutionized the management of chronic myeloid leukemia (CML) and acute promyelocytic leukemia (APL). In 2014, promising results were reported on the use of additional novel targeted agents (e.g. FLT3-ITD and IDH1 inhibitors in AML, ${ }^{60,61}$ ibrutinib and ABT-199 in chronic lymphocytic leukemia (CLL) ${ }^{62,63}$ ). Approvals of many of these novel drugs are based on results of phase II studies with short follow-ups, making the incorporation of these agents into clinical algorithms challenging. Targeted therapies aim at specific mutations, but, since mutations in different genes often coexist, identification of the targeted mutation does not guarantee clinical response to its inhibitor. Even if a good response is achieved, its longevity is not known. Predicting the durability of the benefits of targeted therapies is essential, especially when traditional alternatives are associated with high morbidity and mortality rates. Imagine older adults with $F L T 3$-ITD-positive AML and normal karyotype or fludarabine-resistant CLL, who responded well to quizartinib or ibrutinib consulting you whether to proceed to allo-HCT. Would your advice be different if a patient diagnosed with $\mathrm{Ph}+$ acute lymphocytic leukemia were consulting you after achieving a good response with dasatinib? Firm clinical data will be available to support the selection of specific targeted therapies over allo-HCT only in another few years when follow-ups mature. However, with the pace that novel targeted therapies enter clinical practice, physicians should come up with rational thoughts guiding the best decisions even when available data are premature. Safety profiles of most targeted drugs are good, and thus they become attractive, which challenges the justification for using high-risk procedures like allo-HCT. Availability of novel agents is increasing, and, for those who achieved good responses, prediction of longevity and potential curability is important, especially for the growing population of older adults fit for reducedintensity conditioning allo-HCT.

\section{GENETIC DATA TO SUPPORT THERAPEUTIC DECISIONS IN THE LACK OF CLINICAL EVIDENCE}

Researchers, looking for the very first cancerinitiating event, often concentrate on driver mutations that have the power to transform a normal cell into a malignant one. Yet, some available and effective drugs target mutations which lack the capacity to kick off cancer by themselves but are essential for cancer growth and proliferation. Hematological malignancies vary in their genetic complexity. Not surprisingly, CML and APL, the two success stories of targeted therapies, are characterized by a single dominant oncogenic hybrid protein as their driver event. Pathogenesis of most other hematological malignancies is much more complex, with multiple coexisting combinations of genetic aberrations generating signals stimulating progression of the tumor. In AML, nucleophosmin (NPM1) and FLT3 are the two most commonly mutated genes that have been studied extensively. Both have clinical significance, but biological differences between these mutations should be considered which may help outlining a scheme for translating basic genetic information into rational clinical moves. The most important biological difference between NPM1 and FLT3 mutations lies in the time point where they occur during leukemogenesis. The NPM1 mutation is likely to occur very early during the pathogenesis of leukemia. When injected to immunocompromised mice, NPM1-mutated leukemic cells can generate leukemia. ${ }^{64}$ The NPM1 mutation is stable at diagnosis and relapses and is therefore considered a potential marker for minimal residual disease (MRD). ${ }^{65}$ On the contrary, FLT3-ITD is recognized as a late event, ${ }^{66}$ may be sub-clonal, ${ }^{67}$ and is therefore not reliable as an MRD marker. Indeed, NPM1, but not FLT3-ITD, was recognized as a provisional entity in the 2008 World Health Organization classification of myeloid neoplasms. ${ }^{68}$ However, although FLT3-ITD has no capacity of NPM1 mutation to transform a normal hematopoietic cell into a leukemic one, whenever both mutations coexist, the favorable prognostic effect of NPM1 mutation is overridden by the deleterious prognostic effect of FLT3-ITD. ${ }^{69,70}$ Similarly, t(8:21) is considered to be an early event which yields a well-characterized oncogenic hybrid protein and is stable to be used as an MRD marker.71,72 Yet, concomitant C-kit or ASXL1 mutations which are later events may significantly hamper the prognosis.73,74

The clonal evolution nature of myeloid malignancies $75-77$ is well established. Disease progression may be attributed to a late event resulting in a proliferation and/or survival benefit to a sub-clonal population. Targeted therapies that could block such mutation may lead to a tremendous initial clinical response, but one should bear in mind that such 
response may be short since the therapy is not targeting the primary pathological event. On the contrary, drugs that target the primary event may not be powerful enough to oppose a strong proliferative signal derived from a "second hit" developed in the subpopulation that took over the entire tumor. As there is no reliable mechanism to predict who of the many patients presenting with similar diseases will respond to a specific agent, there is no model forecasting the duration of response in those who achieved a clinically meaningful response.

\section{NEXT-GENERATION SEQUENCING-DO CLINICIANS NEED SO MUCH INFORMATION?}

Genetic profiling of hematological diseases is part of standard assessment. This is particularly significant for acute leukemia, where clinical decisions sometimes depend on the presence or absence of specific mutations. ${ }^{8}$ The development of next-generation sequencing (NGS) techniques allows parallel quantitative sequencing of large numbers of genes at an affordable price.

Next-generation sequencing yields an enormous amount of data which are difficult to interpret, thus requiring skilled bioinformatics expertise. In addition, there is no consensus regarding technical merits of NGS clinical use. 79 Laboratories performing NGS usually provide a comprehensive report including data on multiple genetic aberrations, and in some cases deep sequencing gives information about sub-clonal mutations.

Clinicians are usually familiar with traditional statistical analysis for prognostic evaluation based only on the most common mutation combinations in AML, leaving most patients in a heterogeneous "intermediate-risk" group. ${ }^{80,81}$ There is an unmet need for creating a clinically valid and meaningful "dictionary" for the growing NGS-derived data from private or academic NGS facilities. Clearly, from the clinical perspective not all mutations matter. Mutations with a "weak" prognostic effect are anticipated to be recognized when data from a higher number of analyzed patients become available. Yet, would you recommend your patient spending money on private detailed NGS?

\section{CLINICAL POTENTIAL OF NEXT- GENERATION SEQUENCING}

Genetics is perceived by the majority of hematologists as the most accurate prognostication tool.
Next-generation sequencing is therefore acknowledged for high-level prognostication which, unfortunately, we have limited capacity to deal with. However, NGS is much more than a way of identifying all mutations at a reasonable price. This technique provides an opportunity for dynamic prognostication of patients presenting with myeloid malignancies who may experience leukemic transformation. In recent years, sequential genetic evaluation of the same patient demonstrated the dynamics of progression from MDS to secondary AML. ${ }^{29,75}$ Preliminary studies suggested that accumulation of sub-clonal mutations identified by sequential meticulous genetic follow-up can point out an evolving leukemic transformation. ${ }^{29,82}$ Even mutations which in general have no prognostic value, such as those found in the RAS genes, ${ }^{83}$ may be alarming if they emerge in patients previously tested and found to be negative for these mutations, regardless of R-IPSS classification. ${ }^{84}$ Specific mutations in $A S X L 1, B C O R$, and $I D H 1$ are also associated with a pending leukemic transformation. ${ }^{82,85-87}$ The complexity of leukemia genetics puts an obstacle to revealing the significance of every single mutation for therapeutic decisions. Next-generation sequencing may bring a new dynamic attitude, in which not the presence of a specific mutation but the dynamics and acquisition of new mutations will be of clinical importance. For example, arguments in favor of allo-HCT may sound stronger for patients in whom deterioration toward leukemic transformation can be pre-identified.

\section{CONCLUSIONS}

Techniques for DNA sequencing are rapidly and constantly improving. Clinicians are facing a challenge to employ the new capacities of genetic laboratories to make sure that their patients will benefit most from the ongoing scientific progress. The current review has discussed the contribution of different genetic testing tools to clinical practice. Myeloid malignancies are among the most genetically studied cancerous diseases where genetics is recognized as an important player in diagnosis and/or classification. Our growing understanding of the underlying genetic mechanisms of leukemogenesis may help physicians in daily practice, especially when patients are facing questions where no firm evidence exists to support a specific decision. The explosion of novel targeted agents makes such circumstances frequent. Next-generation sequencing should be considered advantageous, not only because it provides access to a wide 
spectrum of genetic testing for all patients, but also because it may allow recognition of genetics as a dynamic assessment tool of clonal evolution. It may be suggested that in the future, regardless of the presence of a specific mutation, a pending leukemic transformation may be predicted by sequential monitoring of the same patient for newly acquired mutations. Genetics is about to go beyond prognostication and will soon be strongly incorporated into many aspects of clinical evaluation and therapeutic decisions.

\section{REFERENCES}

1. Rowley JD. Identification of a translocation with quinacrine fluorescence in a patient with acute leukemia. Ann Genet 1973;16:109-12.

2. Watts G. Janet Davison Rowley. Lancet 2014;383: 406. Full Text

3. Rowley JD. Letter: A new consistent chromosomal abnormality in chronic myelogenous leukaemia identified by quinacrine fluorescence and Giemsa staining. Nature 1973;243:290-3. Full Text

4. Block M, Jacobson LO, Bethard WF. Preleukemic acute human leukemia. J Am Med Assoc 1953;152: 1018-28. Full Text

5. Bennett JM, Catovsky D, Daniel MT, et al. Proposals for the classification of the myelodysplastic syndromes. Br J Haematol 1982;51:189-99. Full Text

6. Jaffe ES, Harris NL, Stein H, Vardiman JW, eds. World Health Organization Classification of Tumours: Pathology and Genetics of Tumours of Haematopoietic and Lymphoid Tissues. Lyon, France: IARC Press; 2001.

7. Vardiman JW, Thiele J, Arber DA, et al. The 2008 revision of the World Health Organization (WHO) classification of myeloid neoplasms and acute leukemia: rationale and important changes. Blood 2009;114:937-51. Full Text

8. Vardiman J, Hyjek E. World Health Organization classification, evaluation, and genetics of the myeloproliferative neoplasm variants. Hematology Am Soc Hematol Educ Program 2011;2011:250-6. Full Text

9. Foucar K. Myelodysplastic/myeloproliferative neoplasms. Am J Clin Pathol 2009;132:281-9. Full Text

10. Meggendorfer M, Bacher U, Alpermann T, et al. SETBP1 mutations occur in $9 \%$ of MDS/MPN and in $4 \%$ of MPN cases and are strongly associated with atypical CML, monosomy 7, isochromosome i(17) (q10), ASXL1 and CBL mutations. Leukemia 2013;27: 1852-60. Full Text
11. Piazza R, Valletta S, Winkelmann N, et al. Recurrent SETBP1 mutations in atypical chronic myeloid leukemia. Nat Genet 2013;45:18-24. Full Text

12. Kar SA, Jankowska A, Makishima H, et al. Spliceosomal gene mutations are frequent events in the diverse mutational spectrum of chronic myelomonocytic leukemia but largely absent in juvenile myelomonocytic leukemia. Haematologica 2013;98:107-13. Full Text

13. Patnaik MM, Lasho TL, Finke CM, et al. Spliceosome mutations involving $\mathrm{SRSF}_{2}, \mathrm{SF}_{3} \mathrm{~B} 1$, and $\mathrm{U}_{2} \mathrm{AF}_{3} 5$ in chronic myelomonocytic leukemia: prevalence, clinical correlates, and prognostic relevance. Am J Hematol 2013;88:201-6. Full Text

14. Meggendorfer M, Roller A, Haferlach T, et al. SRSF2 mutations in 275 cases with chronic myelomonocytic leukemia (CMML). Blood 2012;120:3080-8. Full Text

15. Maxson JE, Gotlib J, Pollyea DA, et al. Oncogenic $\mathrm{CSF}_{3} \mathrm{R}$ mutations in chronic neutrophilic leukemia and atypical CML. N Engl J Med 2013;368:1781-90. Full Text

16. Pardanani A, Lasho TL, Laborde RR, et al. $\mathrm{CSF}_{3} \mathrm{R}$ T618I is a highly prevalent and specific mutation in chronic neutrophilic leukemia. Leukemia 2013;27: 1870-3. Full Text

17. Nangalia J, Massie CE, Baxter EJ, et al. Somatic CALR mutations in myeloproliferative neoplasms with nonmutated JAK2. N Engl J Med 2013;369: 2391-405. Full Text

18. Tefferi A, Thiele J, Vannucchi AM, Barbui T. An overview on CALR and $\mathrm{CSF}_{3} \mathrm{R}$ mutations and a proposal for revision of WHO diagnostic criteria for myeloproliferative neoplasms. Leukemia 2014;28: 1407-13. Full Text

19. Cazzola M, Kralovics R. From Janus kinase 2 to calreticulin: the clinically relevant genomic landscape of myeloproliferative neoplasms. Blood 2014;123: 3714-19. Full Text

20. Mufti GJ, Bennett JM, Goasguen J, et al. Diagnosis and classification of myelodysplastic syndrome: International Working Group on Morphology of myelodysplastic syndrome (IWGM-MDS) consensus proposals for the definition and enumeration of myeloblasts and ring sideroblasts. Haematologica 2008;93:1712-17. Full Text

21. Malcovati L, Papaemmanuil E, Bowen DT, et al. Clinical significance of $\mathrm{SF}_{3} \mathrm{~B} 1$ mutations in myelodysplastic syndromes and myelodysplastic/myeloproliferative neoplasms. Blood 2011;118:6239-46. Full $\underline{\text { Text }}$ 
22. Cervera N, Itzykson R, Coppin E, et al. Gene mutations differently impact the prognosis of the myelodysplastic and myeloproliferative classes of chronic myelomonocytic leukemia. Am J Hematol 2014; 89:604-9. Full Text

23. Padron E, Abdel-Wahab O. Importance of genetics in the clinical management of chronic myelomonocytic leukemia. J Clin Oncol 2013;31:2374-6. Full Text

24. Shlush LI, Zandi S, Mitchell A, et al. Identification of pre-leukaemic haematopoietic stem cells in acute leukaemia. Nature 2014;506:328-33. Full Text

25. Greenberg $P$, Cox $C$, LeBeau MM, et al. International scoring system for evaluating prognosis in myelodysplastic syndromes. Blood 1997;89:2079-88.

26. Greenberg PL, Tuechler H, Schanz J, et al. Revised international prognostic scoring system for myelodysplastic syndromes. Blood 2012;120:2454-65. Full Text

27. Haferlach T, Nagata Y, Grossmann V, et al. Landscape of genetic lesions in 944 patients with myelodysplastic syndromes. Leukemia 2014;28:241-7. Full $\underline{\text { Text }}$

28. $\mathrm{Xu} \mathrm{L}, \mathrm{Gu} \mathrm{ZH}, \mathrm{Li} \mathrm{Y}$, et al. Genomic landscape of CD34+ hematopoietic cells in myelodysplastic syndrome and gene mutation profiles as prognostic markers. Proc Natl Acad Sci U S A 2014;111:8589-94. Full Text

29. Papaemmanuil E, Gerstung M, Malcovati L, et al. Clinical and biological implications of driver mutations in myelodysplastic syndromes. Blood 2013;122:3616-27; quiz 3699. Full Text

30. Makishima $\mathrm{H}$, Visconte $\mathrm{V}$, Sakaguchi $\mathrm{H}$, et al. Mutations in the spliceosome machinery, a novel and ubiquitous pathway in leukemogenesis. Blood 2012;119:3203-10. Full Text

31. Visconte V, Makishima H, Maciejewski JP, Tiu RV. Emerging roles of the spliceosomal machinery in myelodysplastic syndromes and other hematological disorders. Leukemia 2012;26:2447-54. Full Text

32. Yoshida K, Sanada M, Shiraishi Y, et al. Frequent pathway mutations of splicing machinery in myelodysplasia. Nature 2011;478:64-9. Full Text

33. Schanz J, Tüchler H, Solé F, et al. New comprehensive cytogenetic scoring system for primary myelodysplastic syndromes (MDS) and oligoblastic acute myeloid leukemia after MDS derived from an international database merge. J Clin Oncol 2012;30:820-9. Full Text

34. Such E, Cervera J, Costa D, et al. Cytogenetic risk stratification in chronic myelomonocytic leukemia. Haematologica 2011;96:375-83. Full Text
35. Such E, Germing U, Malcovati L, et al. Development and validation of a prognostic scoring system for patients with chronic myelomonocytic leukemia. Blood 2013;121:3005-15. Full Text

36. Tang G, Zhang L, Fu B, et al. Cytogenetic risk stratification of 417 patients with chronic myelomonocytic leukemia from a single institution. Am J Hematol 2014;89:813-18. Full Text

37. Hussein K, Pardanani AD, Van Dyke DL, Hanson CA, Tefferi A. International Prognostic Scoring Systemindependent cytogenetic risk categorization in primary myelofibrosis. Blood 2010;115:496-9. Full Text

38. Caramazza D, Begna KH, Gangat N, et al. Refined cytogenetic-risk categorization for overall and leukemia-free survival in primary myelofibrosis: a single center study of 433 patients. Leukemia 2011;25:82-8. Full Text

39. Gelsi-Boyer V, Trouplin V, Roquain J, et al. ASXL1 mutation is associated with poor prognosis and acute transformation in chronic myelomonocytic leukaemia. Br J Haematol 2010;151:365-75. Full Text

40. Patnaik MM, Padron E, LaBorde RR, et al. Mayo prognostic model for WHO-defined chronic myelomonocytic leukemia: ASXL1 and spliceosome component mutations and outcomes. Leukemia 2013;27:1504-10. Full Text

41. Vannucchi AM, Lasho TL, Guglielmelli $\mathrm{P}$, et al. Mutations and prognosis in primary myelofibrosis. Leukemia 2013;27:1861-9. Full Text

42. Guglielmelli P, Lasho TL, Rotunno G, et al. The number of prognostically detrimental mutations and prognosis in primary myelofibrosis: an international study of 797 patients. Leukemia 2014;28:1804-10. Full Text

43. Kulasekararaj AG, Smith AE, Mian SA, et al. TP53 mutations in myelodysplastic syndrome are strongly correlated with aberrations of chromosome 5, and correlate with adverse prognosis. Br J Haematol 2013;160:660-72. Full Text

44. Bejar R, Stevenson K, Abdel-Wahab O, et al. Clinical effect of point mutations in myelodysplastic syndromes. N Engl J Med 2011;364:2496-506. Full $\underline{\text { Text }}$

45. Cornelissen JJ, Gratwohl A, Schlenk RF, et al. The European LeukemiaNet AML Working Party consensus statement on allogeneic HSCT for patients with AML in remission: an integrated-risk adapted approach. Nat Rev Clin Oncol 2012;9:579-90. Full $\underline{\text { Text }}$

46. Fenaux P, Mufti GJ, Hellstrom-Lindberg E, et al. Efficacy of azacitidine compared with that of 
conventional care regimens in the treatment of higher-risk myelodysplastic syndromes: a randomised, open-label, phase III study. Lancet Oncol 2009;10:223-32. Full Text

47. Gurion R, Vidal L, Gafter-Gvili A, et al. 5-azacitidine prolongs overall survival in patients with myelodysplastic syndrome--a systematic review and metaanalysis. Haematologica 2010;95:303-10. Full Text

48. List A, Dewald G, Bennett J, et al. Lenalidomide in the myelodysplastic syndrome with chromosome $5 \mathrm{q}$ deletion. N Engl J Med 2006;355:1456-65. Full Text

49. Fenaux P, Giagounidis A, Selleslag D, et al. A randomized phase 3 study of lenalidomide versus placebo in RBC transfusion-dependent patients with Low-/Intermediate-1-risk myelodysplastic syndromes with del5q. Blood 2011;118:3765-76. Full Text

50. Raza A, Reeves JA, Feldman EJ, et al. Phase 2 study of lenalidomide in transfusion-dependent, low-risk, and intermediate-1 risk myelodysplastic syndromes with karyotypes other than deletion $5 q$. Blood 2008; 111:86-93. Full Text

51. Platzbecker U. Who benefits from allogeneic transplantation for myelodysplastic syndromes? New insights. Hematology Am Soc Hematol Educ Program 2013;2013:522-8. Full Text

52. Platzbecker U, Schetelig J, Finke J, et al. Allogeneic hematopoietic cell transplantation in patients age 6070 years with de novo high-risk myelodysplastic syndrome or secondary acute myelogenous leukemia: comparison with patients lacking donors who received azacitidine. Biol Blood Marrow Transplant 2012;18:1415-21. Full Text

53. Koreth J, Pidala J, Perez WS, et al. Role of reducedintensity conditioning allogeneic hematopoietic stemcell transplantation in older patients with de novo myelodysplastic syndromes: an international collaborative decision analysis. J Clin Oncol 2013;31:266270. Full Text

54. Ofran Y, Lazarus HM, Rapoport AP, Rowe JM. Interpreting outcome data in hematopoietic cell transplantation for leukemia: tackling common biases. Bone Marrow Transplant 2014. Accepted for publication.

55. Gratwohl A. The EBMT risk score. Bone Marrow Transplant 2012;47:749-56. Full Text

56. Gratwohl A, Stern M, Brand R, et al. Risk score for outcome after allogeneic hematopoietic stem cell transplantation: a retrospective analysis. Cancer 2009;115:4715-26. Full Text

57. Sorror ML, Sandmaier BM, Storer BE, et al. Comorbidity and disease status based risk stratification of outcomes among patients with acute myeloid leukemia or myelodysplasia receiving allogeneic hematopoietic cell transplantation. J Clin Oncol 2007;25:4246-54. Full Text

58. Bokhari SW, Watson L, Nagra S, et al. Role of HCTcomorbidity index, age and disease status at transplantation in predicting survival and non-relapse mortality in patients with myelodysplasia and leukemia undergoing reduced-intensity-conditioning hemopoeitic progenitor cell transplantation. Bone Marrow Transplant 2012;47:528-34. Full Text

59. Armand P, Kim HT, Logan BR, et al. Validation and refinement of the Disease Risk Index for allogeneic stem cell transplantation. Blood 2014;123:3664-71. Full Text

6o. Cortes JE, Kantarjian H, Foran JM, et al. Phase I study of quizartinib administered daily to patients with relapsed or refractory acute myeloid leukemia irrespective of FMS-like tyrosine kinase 3-internal tandem duplication status. J Clin Oncol 2013;31: 3681-7. Full Text

61. News in Brief: Mutant IDH2 inhibitor looks promising in AML. Cancer Discov 2014;4:6 OF9. Full Text

62. Byrd JC, Furman RR, Coutre SE, et al. Targeting BTK with ibrutinib in relapsed chronic lymphocytic leukemia. N Engl J Med 2013;369:32-42. Full Text

63. Souers AJ, Leverson JD, Boghaert ER, et al. ABT-199, a potent and selective BCL-2 inhibitor, achieves antitumor activity while sparing platelets. Nat Med 2013;19:202-8. Full Text

64. Martelli MP, Pettirossi V, Thiede C, et al. CD34+ cells from AML with mutated NPM1 harbor cytoplasmic mutated nucleophosmin and generate leukemia in immunocompromised mice. Blood 2010;116:390722. Full Text

65. Krönke J, Schlenk RF, Jensen KO, et al. Monitoring of minimal residual disease in NPM1-mutated acute myeloid leukemia: a study from the German-Austrian acute myeloid leukemia study group. J Clin Oncol 2011;29:2709-16. Full Text

66. Kottaridis PD, Gale RE, Linch DC. Flt3 mutations and leukaemia. Br J Haematol 2003;122:523-38. Full Text

67. Shouval R, Shlush LI, Yehudai-Resheff S. Single cell analysis exposes intratumor heterogeneity and suggests that FLT3-ITD is a late event in leukemogenesis. Exp Hematol 2014;42:457-63. Full Text

68. Falini B, Martelli MP, Bolli N, et al. Acute myeloid leukemia with mutated nucleophosmin (NPM1): is it a distinct entity? Blood 2011;117:1109-20. Full Text

69. Schneider F, Hoster E, Unterhalt M, et al. The FLT3ITD mRNA level has a high prognostic impact in NPM1 mutated, but not in NPM1 unmutated, AML 
with a normal karyotype. Blood 2012;119:4383-6. Full Text

70. Linch DC, Hills RK, Burnett AK, Khwaja A, Gale RE. Impact of FLT3(ITD) mutant allele level on relapse risk in intermediate-risk acute myeloid leukemia. Blood 2014;124:273-6. Full Text

71. Yin JA, O'Brien MA, Hills RK, Daly SB, Wheatley K, Burnett AK. Minimal residual disease monitoring by quantitative RT-PCR in core binding factor AML allows risk stratification and predicts relapse: results of the United Kingdom MRC AML-15 trial. Blood 2012;120:2826-35. Full Text

72. Zhu HH, Zhang XH, Qin YZ, et al. MRD-directed risk stratification treatment may improve outcomes of $\mathrm{t}(8 ; 21) \mathrm{AML}$ in the first complete remission: results from the AML05 multicenter trial. Blood 2013;121: 4056-62. Full Text

73. Allen C, Hills RK, Lamb K, et al. The importance of relative mutant level for evaluating impact on outcome of KIT, FLT3 and CBL mutations in corebinding factor acute myeloid leukemia. Leukemia 2013;27:1891-901. Full Text

74. Krauth MT, Eder C, Alpermann T, et al. High number of additional genetic lesions in acute myeloid leukemia with $\mathrm{t}(8 ; 21) / \mathrm{RUNX1}-\mathrm{RUNX1T1}$ : frequency and impact on clinical outcome. Leukemia 2014; 28:1449-58. Full Text

75. Walter MJ, Shen D, Ding L, et al. Clonal architecture of secondary acute myeloid leukemia. N Engl J Med 2012;366:1090-8. Full Text

76. Parkin B, Ouillette P, Li Y, et al. Clonal evolution and devolution after chemotherapy in adult acute myelogenous leukemia. Blood 2013;121:369-77. Full $\underline{\text { Text }}$

77. Krönke J, Bullinger L, Teleanu V, et al. Clonal evolution in relapsed NPM1-mutated acute myeloid leukemia. Blood 2013;122:100-8. Full Text

78. Ofran Y, Rowe JM. Genetic profiling in acute myeloid leukaemia--where are we and what is its role in patient management. Br J Haematol 2013;160:30320. Full Text

79. White BS, DiPersio JF. Genomic tools in acute myeloid leukemia: from the bench to the bedside. Cancer 2014;120:1134-44. Full Text

8o. Patel JP, Gönen M, Figueroa ME, et al. Prognostic relevance of integrated genetic profiling in acute myeloid leukemia. N Engl J Med 2012;366:1079-89. Full Text

81. Grossmann V, Schnittger S, Kohlmann A, et al. A novel hierarchical prognostic model of AML solely based on molecular mutations. Blood 2012;120: 2963-72. Full Text

82. Chen TC, Hou HA, Chou WC, et al. Dynamics of ASXL1 mutation and other associated genetic alterations during disease progression in patients with primary myelodysplastic syndrome. Blood Cancer J 2014;4:e177. Full Text

83. Al-Kali A, Quintás-Cardama A, Luthra R, et al. Prognostic impact of RAS mutations in patients with myelodysplastic syndrome. Am J Hematol 2013;88: 365-9. Full Text

84. Takahashi K, Jabbour E, Wang X, et al. Dynamic acquisition of FLT3 or RAS alterations drive a subset of patients with lower risk MDS to secondary AML. Leukemia 2013;27:2081-3. Full Text

85. Thol F, Friesen I, Damm F, et al. Prognostic significance of ASXL1 mutations in patients with myelodysplastic syndromes. J Clin Oncol 2011;29: 2499-506. Full Text

86. Damm F, Chesnais V, Nagata Y, et al. BCOR and BCORL1 mutations in myelodysplastic syndromes and related disorders. Blood 2013;122:3169-77. Full Text

87. Patnaik MM, Hanson CA, Hodnefield JM, et al. Differential prognostic effect of IDH1 versus IDH2 mutations in myelodysplastic syndromes: a Mayo Clinic study of 277 patients. Leukemia 2012;26:1015. Full Text 